\title{
Regimes espaciais: dinâmica dos homicídios dolosos na cidade de São Paulo entre 2000 e 2008
}

\author{
Marcelo Batista Nery, ${ }^{1}$ Maria Fernanda Tourinho Peres, ${ }^{2}$ \\ Nancy Cardia, ${ }^{1}$ Diego Vicentin ${ }^{1}$ e Sérgio Adorno ${ }^{3}$
}

Como citar Nery MB, Peres MFT, Cardia N, Vicentin D, Adorno S. Regimes espaciais: dinâmica dos homicídios dolosos na cidade de São Paulo entre 2000 e 2008. Rev Panam Salud Publica. 2012;32(6):405-12.

RESUMO Objetivo. Identificar a existência de padrões espaço-temporais na ocorrência de homicídios dolosos no Município de São Paulo (MSP) e discutir o valor analítico de levar em conta tais padrões ao elaborar estudos que tratam da dinâmica e dos fatores associados à incidência dos homicídios dolosos.

Métodos. Realizou-se um estudo ecológico e longitudinal, tendo como unidades de análise 13205 setores censitários e os 96 distritos censitários que congregam esses setores na capital paulista. Foram estudados todos os homicídios dolosos registrados no município entre 2000 e 2008 e calculadas as taxas brutas de homicídios dolosos por 100000 habitantes para cada setor censitário, assim como as taxas bayesianas globais e locais. Para verificar a possibilidade de identificar diferentes padrões de distribuição espacial dos homicídios, foram utilizadas as técnicas BoxMap e o indice de Moran.

Resultados. Não houve uma tendência homogênea e sistemática dos homicídios ao longo da última década na capital paulista. Ao invés disso, foram identificados sete padrões de distribuição espacial, ou seja, sete regimes espaciais, para a ocorrência de homicídios dolosos, considerando as taxas dentro de cada setor censitário e nos setores adjacentes. Esses regimes de distribuição espacial não estavam contidos nos limites dos setores e distritos censitários do município.

Conclusões. Os resultados mostraram a importância de analisar a distribuição espacial dos fenômenos sociais sem restrição de fronteiras politico-administrativas.

Palavras chave Homicídio; distribuição espacial da população; distribuição temporal; sistemas de informação geográfica; Brasil.

Há muito a espacialização do crime tem sido empregada como parte do processo das análises criminais. Entretanto, há apenas poucas décadas surgiram os

\footnotetext{
1 Universidade de São Paulo (USP), Núcleo de Estudos da Violência, São Paulo (SP), Brasil. Correspondência: Marcelo Batista Nery, mbnery@usp.br

2 USP, Faculdade de Medicina, Departamento de Medicina Preventiva, São Paulo (SP), Brasil.

3 USP, Faculdade de Filosofia, Letras e Ciências Humanas, Departamento de Sociologia, São Paulo (SP), Brasil.
}

estudos que utilizam técnicas estatísticas e geoestatíticas para analisar a distribuição espaço-temporal das ocorrências criminais e que ponderam sobre seus escopos explanatórios (1-8).

Nesse sentido, há os estudos que abordam a dinâmica criminal em diferentes localidades pelo viés qualitativo, pela constatação da heterogeneidade de processos sociais no âmbito das cidades, pela avaliação das transformações urbanas ou pelo aprofundamento do conhe- cimento da crise urbana e dos conflitos decorrentes das mais diversas mudanças culturais, econômicas e políticas (9). Igualmente, há estudos que empregam o que se poderia definir, genericamente, como métodos quantitativos $(10,11)$, ou, mais especificamente, métodos multivariados (12-14). Esses estudos procuram explicar a variação das taxas de crime violento e encontrar associações estatísticas entre taxas de crimes e variáveis socioeconômicas dentro de uma mesma 
região ou entre regiões. Além disso, há os que enfoquem microáreas urbanas (15-20).

Em ambos os casos, nos estudos qualitativos e quantitativos, podemos recorrer a diferentes escalas de análise, optando, por exemplo, por observar as ocorrências criminais em escala local (dentro de limites restritos). Essa opção pode ser decorrente do acesso do pesquisador a um grupo, instituição ou relação específicos ou da possibilidade de fazer análises comparativas utilizando os achados na escala local.

Por outro lado, as escalas mais globais podem ser utilizadas quando se pressupõe que a área de estudo: 1) é predominantemente homogênea; 2) apresenta eventos com variação espacial contínua; 3) manifesta em sua totalidade aspectos que podem ser conhecidos a partir das diferentes parcelas que a compõe. A opção pelas abordagens em escalas globais pode ser também resultado de uma necessidade prática: por exemplo, quando o pesquisador tem acesso apenas a dados agregados dos eventos (em unidades de área grandes e heterogêneas), sem a localização precisa das ocorrências de interesse.

No que concerne aos homicídios dolosos - conforme o Código Penal Brasileiro, aqueles em que há intenção de matar - , um fenômeno social complexo e multicausal, qual seria a escala de território mais adequada para se trabalhar? Defendemos aqui a necessidade de desenvolver estudos capazes de integrar a dimensão micro, a fim de evitar conclusões simplistas.

Embora muitos estudos utilizem em suas análises dados de órgãos de saúde, relativos, por exemplo, às causas de morte, sabe-se que a classificação dos órgãos de saúde tem por referência o local de residência da vítima ou o local de registro de morte. A proposição deste artigo é de que as análises dos registros policiais dos homicídios dolosos, que indicam o local de ocorrência do crime, são úteis para identificar padrões espaçotemporais intraurbanos que podem ou não respeitar os limites geográficos das divisões administrativas das cidades.

Sendo assim, o objetivo do presente estudo foi identificar a existência de padrões espaço-temporais na ocorrência de homicídios dolosos no Município de São Paulo (MSP) e discutir o valor analítico de levar em conta tais padrões ao elaborar estudos que tratam da dinâmica e dos os fatores associados à incidência dos homicídios dolosos.

\section{MATERIAIS E MÉTODOS}

Realizou-se um estudo ecológico e longitudinal, tendo como unidade de análise os 13278 setores censitários da capital paulista, com base na divisão censitária de 2000.

\section{Fontes de dados e análise de consistência}

Foram estudados todos os homicídios dolosos registrados no MSP entre 2000 e 2008. É importante diferenciar os homicídios dolosos da categoria "morte por agressão", que expressa óbitos identificados e codificados pelas instituições de saúde, de acordo com a Classificação Internacional de Doenças e Causas de Morte (CID).

Para o presente estudo, as ocorrências registradas pela polícia foram obtidas na base de dados do Sistema de Informações de Ocorrências Criminais (Infocrim) da Secretaria de Segurança Pública de São Paulo (SSP/SP). O Infocrim é um banco de dados do qual é possível extrair informações que permitem identificar, por exemplo, a natureza da ocorrência, o local e a data de ocorrência do crime.

A consistência das informações criminais foi examinada por meio da análise da estabilidade temporal da distribuição espacial das ocorrências criminais agregadas por setor censitário do MSP. Para tanto, inspirados em Vachaud et al. (21), aplicamos dois procedimentos: 1) análise dos desvios entre os valores observados individualmente no espaço e a média desses valores (cálculo da diferença relativa), tomando-se como indicador de estabilidade temporal a constância dos valores ou a presença de pequenas variações entre posições ao longo do tempo; e 2) correlação de Spearman (22), como indicador do grau de variabilidade espacial em diferentes períodos. Embora esse procedimento seja normalmente empregado para estudar a estabilidade temporal da distribuição espacial de características do solo, a metodologia cumpriu o objetivo de indicar os locais com elevada variabilidade espacial dos registros criminais e, consequentemente, de advertir sobre possíveis problemas nas bases de dados e/ou instabilidade espacial.

Também foi necessário mensurar a população para os anos em que não fo- ram realizados os censos demográficos. Os dados demográficos provenientes das informações populacionais do Instituto Brasileiro de Geografia e Estatística (IBGE) de 1991 a 2000 foram utilizados como base para projeções populacionais dos anos intercensitários. Para a projeção populacional por setor censitário foi utilizada a tabela de comparabilidade do IBGE (23). Os números obtidos foram reponderados com base nas projeções populacionais da Fundação Sistema Estadual de Análise de Dados (Seade) para o município; a partir daí, foi aplicada uma taxa decrescente para cada setor censitário (24). Por fim, calculamos as taxas de mortalidade por homicídio doloso (TMHD) por setor.

Como foram utilizados dados secundários da SSP/SP (número de homicídios dolosos por setor censitário) e do IBGE (número de pessoas residentes por setor censitário), que não permitem a identificação da pessoa a que a ocorrência se refere e estão disponíveis mediante solicitação às fontes primárias, é dispensada a necessidade de submissão aos comitês de ética, conforme resolução da Comissão Nacional de Ética em Pesquisa (CONEP).

\section{Análises geoestatísticas}

Calculamos as taxas brutas de homicídios dolosos por 100000 habitantes para cada setor censitário entre 2000 e 2008. Para minimizar o problema da instabilidade das taxas em pequenas áreas e o grande número de setores com taxa zero (o que ocorre quando são utilizadas as taxas brutas), foram estimadas taxas bayesianas globais e locais (25-27). Grosso modo, as taxas bayesianas são mensurações que consideram efeitos espaciais, ou seja, em uma área de estudo formada por diversas unidades territoriais, estima-se a taxa de uma localidade levando em consideração os dados da própria unidade e de todo o conjunto de unidades (taxa global), ou estima-se a taxa de uma localidade levando em consideração os dados da própria unidade e os dados das unidades vizinhas (taxa local). A estratégia de levar em conta tanto os dados da área bem como os da região e de sua vizinhança (unidades territoriais fronteiriças) torna as taxas menos instáveis.

Após observar o resultado do cálculo das medidas de dispersão e tendência central e do coeficiente de correlação de 
Spearman, das taxas brutas de homicídios, das taxas bayesianas locais e globais e do número absoluto de ocorrências, constatamos que as taxas bayesianas locais eram o melhor indicador das TMHD para esta análise. Os valores mínimos e máximos, as medidas centrais e de dispersão e a evolução das taxas podem ser observados na tabela 1. As estimativas bayesianas locais, mesmo tendendo a homogeneizar as taxas, não eliminam a possibilidade de haver setores com taxas muito elevadas, como demonstram os valores máximos de 2003, 2005 e 2007, especialmente. Nesses anos, observando a taxa bayesiana do MSP (taxa média do município por 100 mil habitantes) e a média das taxas para o conjunto de setores censitários da capital, notamos que os valores extremos influenciam a evolução dessas médias.

Diante disso, a presença de outliers entre os setores censitários foi analisada tomando-se como referência as taxas bayesianas locais. Para identificar esses valores considerados excepcionais ou desviantes, foi utilizado um teste estatístico direcionado a essa verificação, o teste $\mathrm{Q}$ de Dixon (28-30).

A concentração de casos em poucos setores embasou o uso de métodos capazes de gerar medidas de auto-correlação espacial e local (31). Privilegiamos, nesse estágio, a análise dos dados espaciais para examinar como se dá a distribuição espacial dessas ocorrências. Para verificar a possibilidade de identificar a existência de diferentes padrões de distribuição espacial dos homicídios, foram utilizadas técnicas BoxMap e o índice de Moran, bem como testada a significância deste último.

O BoxMap permite retratar o comportamento das taxas de uma área em comparação ao comportamento das taxas de seus vizinhos. O índice de Moran pro- duz uma medida de associação espacial existente no conjunto dos dados, global e local. De forma geral, objetivamos, com o uso dessas técnicas: identificar a existência de uma tendência geral de agrupamento das taxas de homicídios dolosos, de uma estrutura de correlação espacial e, sendo identificada a existência de dependência espacial, expor como os valores estão correlacionados no espaço.

\section{RESULTADOS}

Entre 2000 e 2008 houve uma queda de $73 \%$ nas taxas de homicídios dolosos no MSP, de 55,7 para 14,9 por 100000 habitantes (dados da SSP/SP). Entretanto, a análise dos dados por setor censitário mostra que a queda não foi homogênea e sistemática.

Seguindo os procedimentos citados anteriormente, quatro casos foram identificados como discrepantes. Além disso, 69 setores apresentavam a ausência de informações completas que permitissem calcular as taxas de homicídios. No total, 73 setores censitários foram eliminados; portanto, as análises se referem a 13205 unidades de área.

Como esperado, o BoxMap revelou a presença de padrões de distribuição espaciais, ou seja, de regimes espaciais, para a ocorrência de homicídios dolosos que não estavam contidos nos limites dos setores censitários no MSP. O cálculo dos índices de Moran e a análise da significância não permitiram rejeitar a hipótese de auto-correlação espacial entre as taxas de homicídios dolosos por setor censitário. Essa evidência exigiu uma caracterização mais detalhada desses regimes espaciais.

O exame dos regimes permitiu identificar sub-regiões que apresentavam um padrão espacial próprio, que se manteve constante na maior parte do período de
9 anos do estudo. O aprofundamento da análise revelou que os quatro tipos de regimes espaciais inicialmente identificados por meio do BoxMap não eram suficientes para explicar o contexto encontrado na capital paulista. Verificamos, então, a pertinência de adicionar no cômputo dos regimes espaciais o número absoluto de homicídios dolosos para o MSP. Dessa forma, seria possível diferenciar os locais cujas estimativas apontavam altas taxas de homicídios dolosos (altas em relação ao valor da média dos setores censitários e da média dos seus vizinhos), mas onde não havia registro de homicídio doloso, segundos dados policiais.

A análise espacial utilizando as taxas bayesianas locais e os números absolutos de homicídios revelou a presença de sete tipos de regimes, cada qual apresentando um padrão distinto de distribuição e concentração. Os sete regimes são descritos a seguir.

- Setor censitário com altas taxas de homicídios dolosos em vizinhança com altas taxas, mas sem nenhuma ocorrência registrada no período 2000-08: alto0 (AA0). As taxas bayesianas locais levam em conta tanto os números do setor censitário como de sua vizinhança. Portanto, foram identificados lugares que não registraram nenhum homicídio doloso e, mesmo assim, devido às taxas dos setores que os circundam, apresentaram altas taxas de homicídios.

- Altas taxas de homicídios dolosos em uma vizinhança com altas taxas: alto (AA).

- Nenhum homicídio doloso registrado no período 2000-08: baixo0 (BB0). Os setores sem homicídio doloso registrado, mas que apresentaram altas taxas de homicídios dolosos em uma

TABELA 1. Estatísticas das taxas bayesianas locais relativas a homicídios dolosos para os setores censitários e taxa bayesiana do Município de São Paulo (SP), Brasil, 2000 a 2008

\begin{tabular}{|c|c|c|c|c|c|c|c|c|c|}
\hline Ano & 2000 & 2001 & 2002 & 2003 & 2004 & 2005 & 2006 & 2007 & 2008 \\
\hline Valor mínimo & 0 & 0 & 0 & 0 & 0 & 0 & 0 & 0 & 0 \\
\hline Valor máximo & 77 & 105 & 177 & 965 & 253 & 444 & 190 & 693 & 167 \\
\hline $\begin{array}{l}\text { Taxa bayesiana do MSP por } \\
100000 \text { habitantes }\end{array}$ & 64 & 62 & 58 & 62 & 45 & 42 & 25 & 32 & 18 \\
\hline Média & 0,7 & 0,6 & 0,6 & 0,7 & 0,5 & 0,4 & 0,2 & 0,3 & 0,2 \\
\hline Mediana & 0,3 & 0,3 & 0,3 & 0,2 & 0,2 & 0,1 & 0,0 & 0,0 & 0,0 \\
\hline Desvio padrão & 1,6 & 1,8 & 2,4 & 8,8 & 2,5 & 6,1 & 2,6 & 8,1 & 2,1 \\
\hline Intervalo interquartil (25-75\%) & 0,6 & 0,7 & 0,6 & 0,6 & 0,5 & 0,3 & 0,2 & 0,2 & 0,1 \\
\hline
\end{tabular}

Fonte: Secretaria de Segurança Pública do Estado de São Paulo, Sistema de Informações de Ocorrências Criminais (Infocrim). 
vizinhança com altas taxas, foram incluídos em AA0, e não nesta categoria.

- Baixas taxas de homicídios dolosos em uma vizinhança com baixas taxas: baixo (BB).

- Baixas taxas de homicídios dolosos em uma vizinhança com altas taxas: baixo-alto (BA).

- Altas taxas de homicídios dolosos em uma vizinhança com baixas taxas: alto-baixo (AB).

- Setores que não repetiram exatamente o mesmo padrão em ao menos 5 dos 9 anos observados: sem padrão definido (SP).

A distribuição dos setores no território do MSP, segundo os sete padrões identificados, pode ser observada na figura 1 .

Utilizando como base de análise o distrito censitário (áreas com limites territoriais determinados por lei municipal, que agregam os setores censitários, existindo no MSP 96 distritos), observamos que a distribuição dos sete regimes identificados não acompanha os limites geográficos desses distritos:

- Os 129 setores censitários caracterizados como AA0 estavam localizados em 45 distritos censitários.

- Os 1105 setores censitários caracterizados como AA estavam distribuídos em 73 dos 96 distritos censitários.

- Os 3984 setores censitários caracterizados como BB0 estavam espalhados em 94 dos 96 distritos da capital; as exceções são Engenheiro Marsilac e Socorro.

- Os 5669 setores censitários caracterizados como BB estavam distribuídos em quase todos os distritos censitários, com exceção de Engenheiro Marsilac.

- Os 471 setores censitários caracterizados como BA estavam distribuídos em 70 dos 96 distritos censitários paulistas.

- Os 15 setores censitários caracterizados como $\mathrm{AB}$ estavam localizados em 14 distritos censitários: Cangaíba, Capão Redondo, Cidade Tiradentes, Itaim Paulista, Jabaquara, Jaraguá, Jardim Helena, Parque do Carmo, Penha, Raposo Tavares, Sapopemba, Tatuapé, Vila Sonia e dois em Pedreira.

- Os 1832 setores caracterizados como SP distribuem-se por 89 distritos censitários, exceção feita a Água Rasa, Mandaqui, Moema, Perdizes, Tucuruvi, Vila Formosa e Vila Mariana.
A existência de especificidades nos regimes espaciais deve ser avaliada. Cerca de $20 \%$ dos 129 setores com altas taxas de homicídios que estavam em uma vizinhança também com altas taxas, mas com nenhuma ocorrência registrada em todo o período analisado (AA0), eram aglomerados subnormais (conjuntos habitacionais, favelas e assemelhados, segundo classificação do IBGE). Os setores com baixas taxas em vizinhança com altas taxas (BA) representaram apenas $3,6 \%$ do total, enquanto os setores com altas taxas, tendo no entorno setores com baixas taxas $(\mathrm{AB})$, foram ainda mais raros, apenas $0,1 \%$, sendo que nenhum setor sustentou essa última condição por todo o período (2000-2008). Diante dessa verificação, duas hipóteses podem ser levantas: 1) uma vizinhança não sustenta baixas taxas de homicídios tendo em seu centro um setor com altas taxas de violência e 2) um setor com altas taxas de homicídio doloso em uma vizinhança de baixas taxas tenderá a apresentar uma queda nessas taxas de homicídio.

Outra importante característica desses regimes espaciais é apresentarem uma evolução específica das TMHD para cada um dos regimes identificados, conforme figura 2.

Esses padrões de distribuição apresentaram estabilidade ao longo do período estudado. Por outro lado, alguns setores censitários sofreram alteração em um dos anos, mas mantiveram-se estáveis nos anos seguintes. Deve-se avaliar a possibilidade de tratar esses setores como outliers dentro do regime no qual foram classificados. Nesse caso, não podem ser utilizados em análises que buscam estabelecer associações. Considerando, portanto, a mudança de regime, a distribuição dos setores pode ser visualizada (figura 3 ). Têm-se assim setores censitários com regimes espaciais:

- Com altas taxas de homicídios dolosos, em qualquer tipo de vizinhança e que, a partir de certo momento, passam a registrar baixas taxas em qualquer tipo de vizinhança (AAAB <ano> BBBA), mantendo-se assim até o fim do período.

- Com baixas taxas de homicídios dolosos em qualquer tipo de vizinhança e que, a partir de certo momento, passam a registrar altas taxas em qualquer tipo de vizinhança (BBBA < ano $>A A A B)$ ao longo de todo o período.
As figuras 1 e 3 permitem constatar que os padrões espaciais identificados não respeitam a divisão administrativa distrital, segundo a qual o MSP está dividido em 96 distritos censitários.

Nos distritos de Jardim São Luis (102 setores) e Jardim Ângela (83 setores), distritos periféricos da zona sul da capital paulista, quase um terço setores censitários apresentaram padrão de altas taxas de homicídio em uma vizinhança também com altas taxas (AA). No centro da capital, onde se poderia imaginar um padrão que refletisse um menor grau de violência, comparado à condição dos distritos da periferia do município, encontramos um quadro ainda mais grave. Distritos da região central da capital, como Bom Retiro, Sé e Brás, respectivamente, tiveram $43 \%, 52 \%$ e $58 \%$ de seus setores com altas taxas de homicídios em uma vizinhança com altas taxas ao longo do período (AA). Por outro lado, Saúde, Vila Mariana, Moema (que compõem a Subprefeitura da Vila Mariana), unidos aos distritos Itaim Bibi e Jardim Paulista, formam uma área contígua na porção centro-sul da capital que se destaca por possuir um elevado número de setores censitários sem qualquer registro de homicídios dolosos no período entre 2000 e 2008 (BB0).

\section{DISCUSSÃO}

Embora estudos recentes formulem hipóteses para explicar a dinâmica dos homicídios dolosos e sua expressiva queda em São Paulo nos últimos anos (32-41), não há ainda consenso quanto aos seus condicionantes ou quanto à sua dinâmica. Os resultados alcançados nesses estudos mostram que existem fatores (econômicos, estruturais, demográficos, sociais, entre outros) invariavelmente associados com altas taxas de homicídios, ao passo que outros fatores apresentam comportamento mais volátil (4).

Desenvolvemos dois estudos complementares a esses trabalhos anteriores. Em uma primeira etapa, elaboramos um estudo ecológico de série temporal que descreveu a evolução dos homicídios em São Paulo (32), empregando taxas de mortes por agressões. No presente trabalho, identificamos os padrões espaço-temporais dos homicídios dolosos, evidenciando o valor analítico de localizá-los e caracterizá-los, também para compreender as causas de sua queda. 


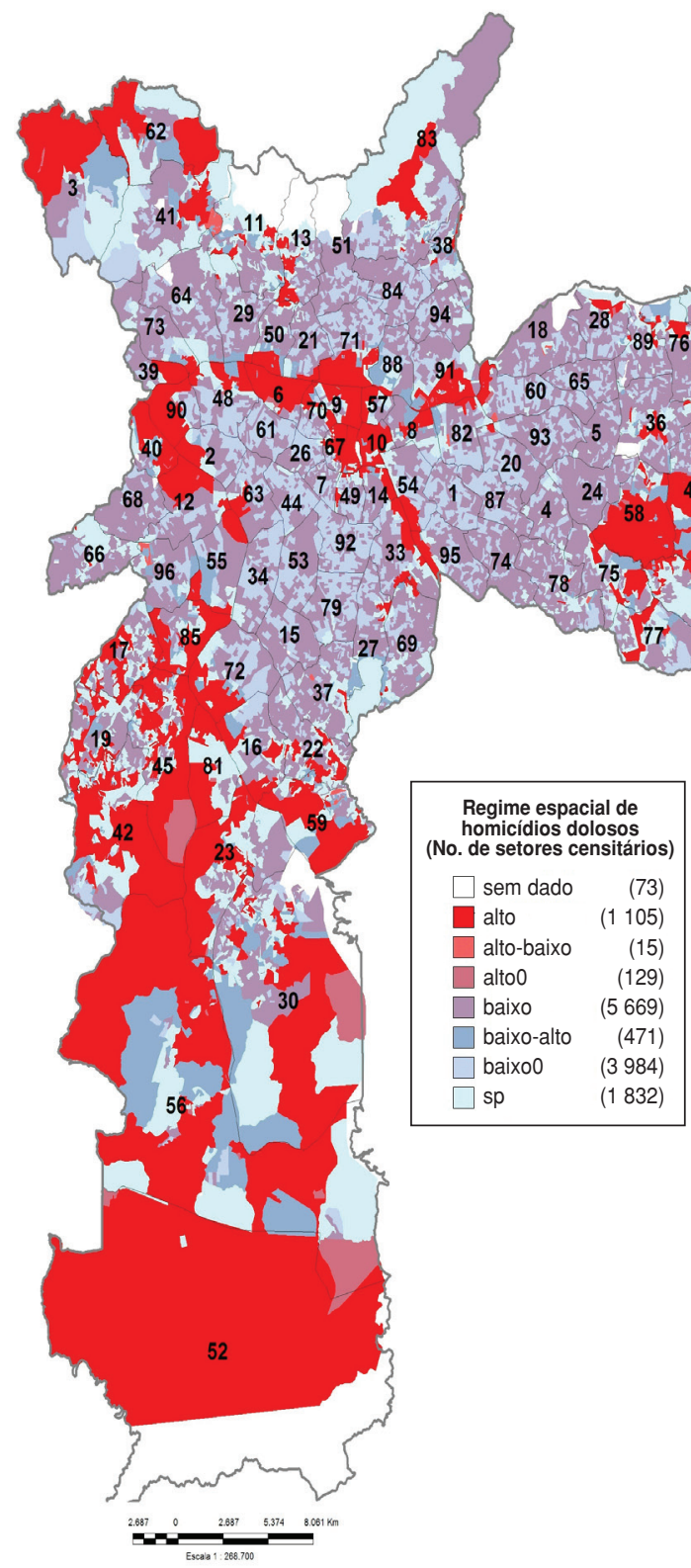

\begin{tabular}{|r|l|}
\hline \multicolumn{1}{|l|}{ Código } & Distrito \\
\hline 1 & AGUA RASA \\
\hline 2 & ALTO DE PINHEIROS \\
\hline 3 & ANHANGUERA \\
\hline 4 & ARICANDUVA \\
\hline 5 & ARTUR ALVIM \\
\hline 6 & BARRA FUNDA \\
\hline 7 & BELA VISTA \\
\hline 8 & BELEM \\
\hline 9 & BOM RETIRO \\
\hline 10 & BRAS \\
\hline 11 & BRASILANDIA \\
\hline 12 & BUTANTA \\
\hline 13 & CACHOEIRINHA \\
\hline 14 & CAMBUCI \\
\hline 15 & CAMPO BELO \\
\hline 16 & CAMPO GRANDE \\
\hline 17 & CAMPO LIMPO \\
\hline 18 & CANGAIBA \\
\hline 19 & CAPAO REDONDO \\
\hline 20 & CARRAO \\
\hline 21 & CASA VERDE \\
\hline 22 & CIDADE ADEMAR \\
\hline 23 & CIDADE DUTRA \\
\hline 24 & CIDADE LIDER \\
\hline 25 & CIDADE TIRADENTES \\
\hline 26 & CONSOLACAO \\
\hline 27 & CURSINO \\
\hline 28 & ERMELINO MATARAZZO \\
\hline 29 & FREGUESIA DO O \\
\hline 30 & GRAJAU \\
\hline 31 & GUAIANAZES \\
\hline 32 & IGUATEMI \\
\hline 33 & IPIRANGA \\
\hline & \\
\hline
\end{tabular}

\begin{tabular}{|c|c|c|c|}
\hline Código & Distrito & Código & Distrito \\
\hline 33 & IPIRANGA & 65 & PONTE RASA \\
\hline 34 & ITAIM BIBI & 66 & RAPOSO TAVARES \\
\hline 35 & ITAIM PAULISTA & 67 & REPUBLICA \\
\hline 36 & ITAQUERA & 68 & RIO PEQUENO \\
\hline 37 & JABAQUARA & 69 & SACOMA \\
\hline 38 & JACANA & 70 & SANTA CECILIA \\
\hline 39 & JAGUARA & 71 & SANTANA \\
\hline 40 & JAGUARE & 72 & SANTO AMARO \\
\hline 41 & JARAGUA & 73 & SAO DOMINGOS \\
\hline 42 & JARDIM ANGELA & 74 & SAO LUCAS \\
\hline 43 & JARDIM HELENA & 75 & SAO MATEUS \\
\hline 44 & JARDIM PAULISTA & 76 & SAO MIGUEL \\
\hline 45 & JARDIM SAO LUIS & 77 & SAO RAFAEL \\
\hline 46 & JOSE BONIFACIO & 78 & SAPOPEMBA \\
\hline 47 & LAJEADO & 79 & SAUDE \\
\hline 48 & LAPA & 80 & $\mathrm{SE}$ \\
\hline 49 & LIBERDADE & 81 & SOCORRO \\
\hline 50 & LIMAO & 82 & TATUAPE \\
\hline 51 & MANDAQUI & 83 & TREMEMBE \\
\hline 52 & MARSILAC & 84 & TUCURUVI \\
\hline 53 & MOEMA & 85 & VILA ANDRADE \\
\hline 54 & MOOCA & 86 & VILA CURUCA \\
\hline 55 & MORUMBI & 87 & VILA FORMOSA \\
\hline 56 & PARELHEIROS & 88 & VILA GUILHERME \\
\hline 57 & PARI & 89 & VILA JACUI \\
\hline 58 & PARQUE DO CARMO & 90 & VILA LEOPOLDINA \\
\hline 59 & PEDREIRA & 91 & VILA MARIA \\
\hline 60 & PENHA & 92 & VILA MARIANA \\
\hline 61 & PERDIZES & 93 & VILA MATILDE \\
\hline 62 & PERUS & 94 & VILA MEDEIROS \\
\hline 63 & PINHEIROS & 95 & VILA PRUDENTE \\
\hline 64 & PIRITUBA & 96 & VILA SONIA \\
\hline 65 & PONTE RASA & & \\
\hline
\end{tabular}

Fonte: Núcleo de Estudos da Violência da Universidade de São Paulo.

Os dados agregados apenas não permitem caracterizar a heterogeneidade da evolução dos homicídios dolosos ocorridos no território paulistano. Há, assim, o desafio de compreender a distribuição espacial dos fenômenos sociais em uma condição na qual a maior parte das informações é oriunda de levantamentos realizados por órgãos públicos que agregam os dados em delimitações político-administrativas, sem atentar para a sua heterogeneidade interna e sem uma validação analítica consistente da forma como as fronteiras foram determinadas.

Devemos observar ainda que, na definição da área de análise e de sua extensão, vários problemas podem surgir. Do ponto de vista quantitativo, um dos principais problemas de empregar dados agregados por áreas é que as estimativas e os resultados dos testes podem ser distorcidos. A agregação tende a aumentar a correlação entre as variáveis e a reduzir as flutuações estatísticas (42). Do mesmo modo, ao estabelecer relações de associação entre diferentes mensurações, lembramos a possibilidade de incorrer 
FIGURA 2. Evolução das taxas médias de homicídios dolosos por 100000 habitantes segundo regime espacial ${ }^{\mathrm{a}}$, São Paulo (SP), Brasil, 2000 a 2008

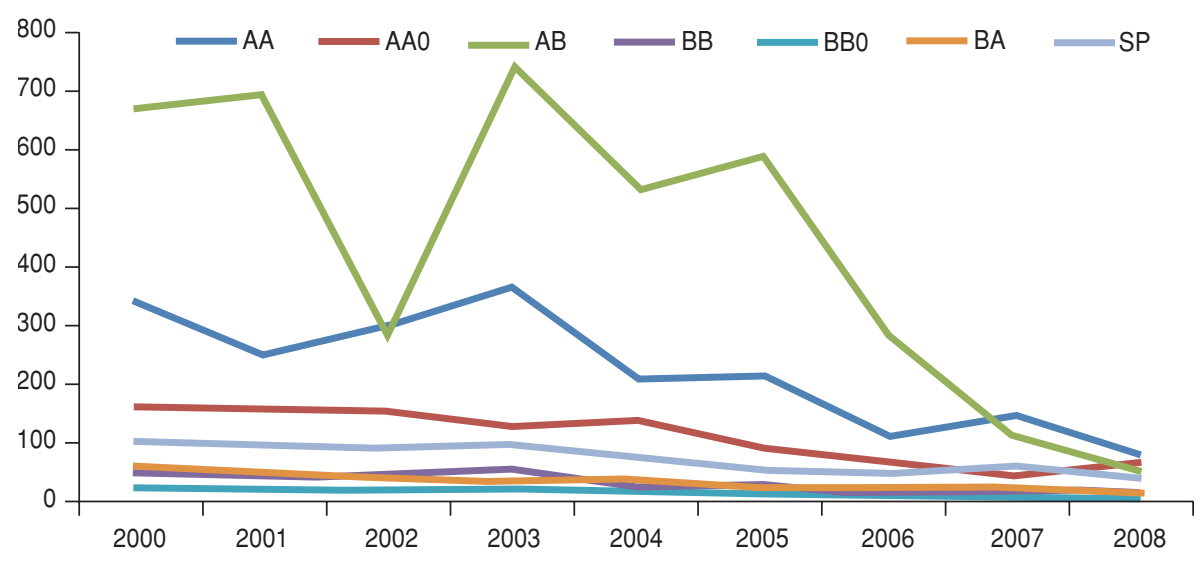

Fonte: Núcleo de Estudos da Violência da Universidade de São Paulo.

a Regimes espaciais: $A A=$ altas taxas de homicídio em vizinhança com altas taxas; $A A 0$ = altas taxas de homicídio em vizinhança com altas taxas, mas sem ocorrência registrada no período; $A B$ = altas taxas em vizinhança com baixas taxas; $\mathrm{BB}$ = baixas taxas de homicídio em vizinhança com baixas taxas; $\mathrm{BB} 0=$ nenhum homicídio registrado no período; $\mathrm{BA}=$ baixas taxas em vizinhança com altas taxas; $\mathrm{SP}=$ sem padrão definido, ou seja, setores que não repetem exatamente 0 mesmo padrão em ao menos 5 dos 9 anos observados. no fenômeno denominado "falácia ecológica" (43-45), que ocorre quando medidas agregadas são inadequadamente utilizadas para inferências individuais (46). Assim, não é possível traduzir para o nível individual as conclusões obtidas com base na análise de dados agregados. Do ponto de vista mais qualitativo, o primeiro ponto a observar é que existem variáveis envolvidas na definição de uma área de estudo (47), entre as quais se destacam os conflitos reais e latentes entre atores sociais e a multiplicidade de interesses envolvidos nesses conflitos.

É possível lidar com essas questões. Podem-se elaborar formas de definir a melhor área de estudo, com base nas características gerais de instituições, organizações, cidades ou partidos políticos, por exemplo; ou, ainda, com base no vínculo entre as pessoas em um território, nas atividades sociais e econômicas comuns (48), ou na união de determina-

FIGURA 3. Setores censitários com abruptas mudanças de padrão de homicídios dolosos, São Paulo (SP), Brasil, 2000 a 2008
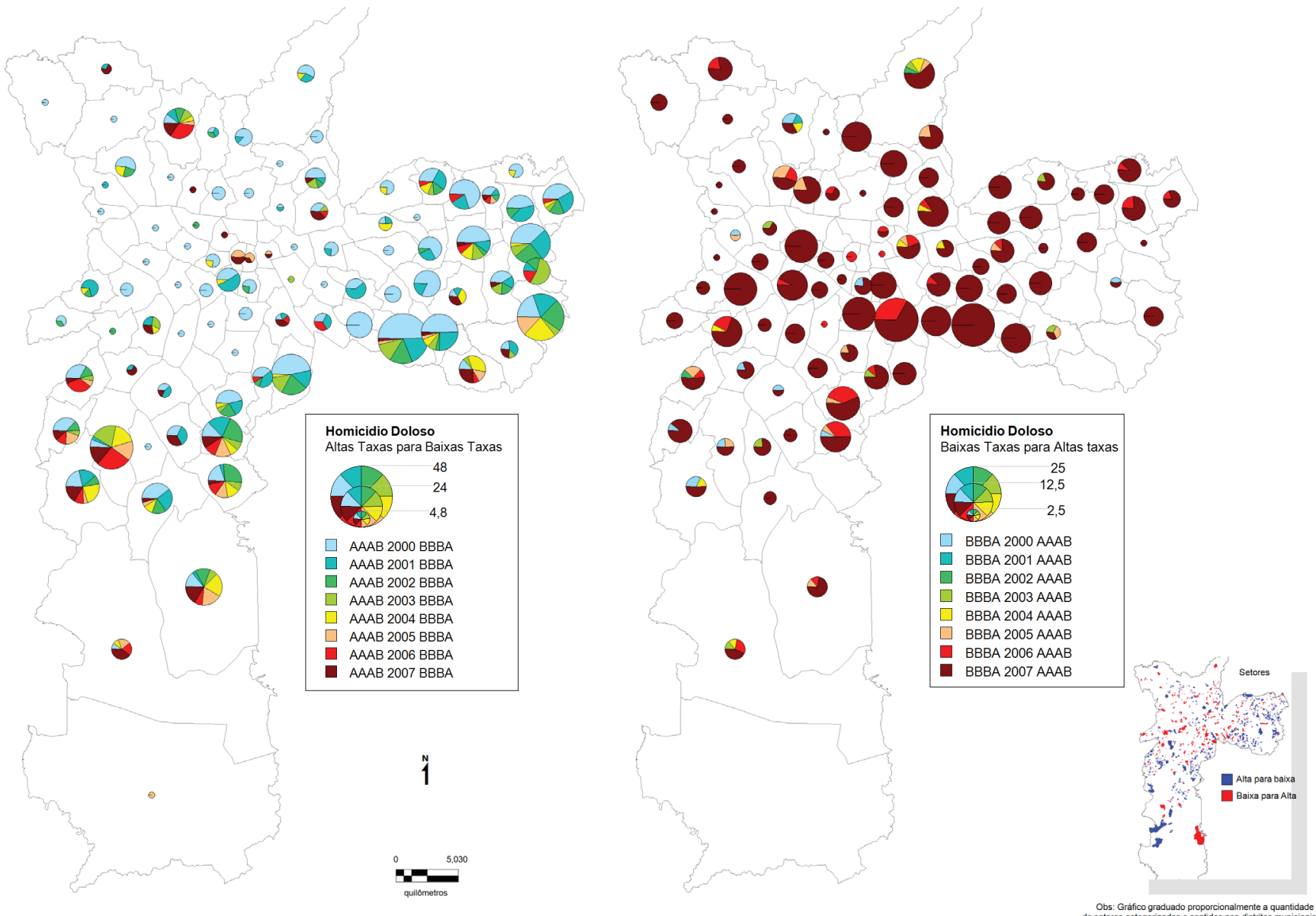

Fonte: Núcleo de Estudos da Violência da Universidade de São Paulo. 
dos tipos de atores que, por meio de sua organização, podem partilhar ou tentar partilhar o poder no território (49).

Concluímos, com base na análise dos dados apresentados, que os setores censitários do MSP apresentam padrões de agregação territoriais específicos que não correspondem a uma divisão político-administrativa pré-estabelecida. Nesse sentido, a principal contribuição deste trabalho é identificar padrões espaciais que se estruturam em torno das distintas dinâmicas de homicídios, que podem ser utilizados como referência em estudos que pretendam identificar os condicionantes dos homicídios em um dado território. Sugere-se a utilização dos dados a partir da forma mais desagregada disponível (os setores censitários servem como exemplo), aplicando a esses dados técnicas quantitativas para obter áreas mais agregadas que preservem o fenômeno estudado em suas singularidades. Essa metodologia pode orientar estudos epidemiológicos e influenciar políticas públicas multissetoriais inteligentes $\mathrm{e}$ articuladas - com ênfase nas ações preventivas, territorialmente determinadas.

Agradecimentos. Os autores agradecem às seguintes instituições e pessoas: Coordenadoria de Análise e Planejamento da Secretaria de Segurança de São Paulo e Instituto Brasileiro de Geografia e Estatística pelas valiosas informações, bem como Cristiane De Leo Ballanotti, Rafael Werneck Cinoto, Mariana Thorstensen Possas e Flávia de Fonseca Feitosa pelas importantes sugestões.

\section{REFERÊNCIAS}

1. Land KC, McCall PL, Cohen L. Structural covariates of homicide rates: are there any invariances across time and social space? Am J Sociol. 1990;95(4):922-63.

2. McCall PL, Land KC, Parker KF. An empirical assessment of what we know about structural covariates of homicide rates: a return to a classic 20 years later. Homicide Stud. 2010;14(3):219-43.

3. Pridemore WA, Trent CLS. Do the invariant findings of Land, McCall, and Cohen generalize to cross-national studies of social structure and homicide? Homicide Stud. 2010;14(3):296-335.

4. Beato CCF. Determinantes da criminalidade em Minas Gerais. Rev Bras Ci Soc. 1998;13(37):74-87.

5. Bailey TC. Review of statistical spatial analysis in GIS. Em: Fotheringham S, Rogerson P, eds. Spatial analysis and GIS. London: Taylor \& Francis; 1994. Pp. 13-44.

6. Câmara G, Yi JRL, Felgueiras C, Moneiro AA. Análise espacial da distribuição e dinâmica da violência na cidade de São Paulo entre os anos de 1996 e 1999. São José dos Campos: INPE; 2000.

7. Harries K. Mapping crime: principle and practice. Washington, DC: US Department of Justice, Office of Justice Programs; 1999.

8. Messner S, Anselin L, Baller R, Hawkins D, Deane G, Tolnay S. The spatial patterning of county homicide rates: an application of exploratory spatial data analysis. J Quant Criminol. 1999;15(4):423-50.

9. Zaluar A. Violência e crime. Em: Miceli S. O que ler na ciência social brasileira (1970-1995). São Paulo: Sumaré; 1999.

10. Schuerman L, Kobrin S. Community careers in crime. Em: Tonry M, Morris N, eds. Crime and justice. Chicago: The University of Chicago Press; 1986. Pp. 67-100.

11. Wolfgang ME, Ferracuti F. The subculture of violence. Londres: Tavistock; 1967.

12. Carvalho MS, Cruz OG, Nobre FF. Perfil de risco: método multivariado de classificação sócio-econômica de microáreas urbanas os setores censitários da região metropolitana do Rio de Janeiro. Cad Saude Publica. 1997;13(4):635-45.
13. Gawryszewski VP, Costa LS. Homicídios e desigualdades sociais no Município de São Paulo. Rev Saude Publica. 2005;39(2):191-7.

14. Villaça F. Espaço intra-urbano no Brasil. São Paulo: FAPESP; 1998

15. Beato CCF, Assunção RM, Reis IA, Marinho FC, Bráulio FAZ, Almeida MC. Conglomerados de homicídios e o tráfico de drogas em Belo Horizonte. Cad Saude Publica. 2001;17(5):1163-71.

16. Beato CCF, Reis IA. Desigualdade, desenvolvimento sócio-econômico e crime. Em: Henriques R, ed. Desigualdade e pobreza no Brasil. Rio de Janeiro: IPEA; 1999. Pp. 385-404.

17. Carneiro LP. Determinantes do crime na América Latina: Rio de Janeiro e São Paulo [relatório de pesquisa]. São Paulo: USP; 1999.

18. Ferreira FF. Modelo espacial de verificação do impacto do Programa de Observação e Vigilância da Polícia Militar de Belo Horizonte (MG) [dissertação]. Belo Horizonte: Universidade Federal de Minas Gerais; 2001.

19. Lima MLC, Ximenes RAA, Souza ER, Luna CF, Albuquerque MFPM. Spatial analysis of socioeconomic determinants of homicide in Brazil. Rev Saude Publica. 2005;39(2):176-82.

20. Pezzin LE. Criminalidade urbana e crise econômica: o caso de São Paulo. São Paulo: IPE/ USP; 1987.

21. Vachaud G, Passerat de Silane A, Balabanis P, Vauclin M. Temporal stability of spatially measured soil water probability density function. Soil Sci Soc America J. 1985;49:822-7.

22. Campos H. Estatística experimental não-paramétrica. Piracicaba: ESALQ/FEALQ; 1983.

23. Instituto Brasileiro de Geografia e Estatística (IBGE). Tabela de comparabilidade: setores censitários 1991-1996-2000. São Paulo: IBGE; 2000.

24. Qasim SR. Wastewater treatment plants: planning, design, and operation. Nova Iorque: Holt, Rinehart and Winston/CBS College Publishing; 1985.

25. Anselin L, Syabri I, Ko Y. GeoDa: an introduction to spatial data analysis. Geogr Anal. 2005;38:5-22.

26. Assunção RM, Reis EA. A new proposal to adjust Moran's I for population density. Stat Med. 1999;18(16):2147-62.
27. Marshall RJ. Mapping disease and mortality rates using empirical Bayes estimators. J Roy Stat Soc C-App. 1991;40(2):283-94.

28. Dixon WJ. Ratios involving extreme values. Ann Math Stat. 1951;22(1):68-78.

29. Gómez-Biedma S, Vivó M, Soria E. Pruebas de significación en bioestadística. Rev Diagn Biol. 2001;50(4):207-18.

30. Rorabacher DB. Statistical treatment for rejection of deviant values: critical values of Dixon's " $\mathrm{Q}$ " parameter and related and related subrange ratios at the $95 \%$ confidence level. Anal Chem. 1991;63(2):139-46.

31. Anselin L, Bera A. Spatial dependence in linear regression models with an introduction to spatial econometrics. Em: Ullah A, Giles D, eds. Handbook of applied economic statistics. Nova Iorque: Marcel Dekker; 1998. Pp. 237-89.

32. Peres MFT, Vicentin D, Nery MB, Lima RS, Souza ER, Cerda M, et al. Queda dos homicídios em São Paulo, Brasil: uma análise descritiva. Rev Panam Salud Publica. 2011;29(1):1726.

33. Adorno S. Análise de pesquisa - segurança. DNA Paulistano. São Paulo: Publifolha; 2009. Pp. 359-62.

34. Biderman C, Mello JMP, Schneider AA. Dry laws and homicides: evidence from the São Paulo metropolitan area. The Economic J. 2010;120(543):157-82.

35. Fonzar UJV. Análise espacial da mortalidade por causas externas no município de Maringá, Estado do Paraná, 1999 a 2001. Acta Sci Health Sci. 2008;30(2):145-54.

36. Hartung GC. Ensaios em Demografia e Criminalidade [tese]. Rio de Janeiro: EPGE; 2009.

37. McCall PL, Parker KF, MacDonald JM. The dynamic relationship between homicide rates and social, economic, and political factors from 1970 to 2000 . Soc Scie Res. 2008;37(3):721-35.

38. Scripilliti EAO. Crimes nos municípios paulistas: um estudo acerca dos condicionantes sócio-econômicos e demográficos que contribuem para maior criminalidade e quais os efeitos das diferentes políticas municipais de segurança para o combate à criminalidade [dissertação]. São Paulo: USP; 2006. 
39. Waiselfisz JJ. Mapa da violência, 2010: anatomia dos homicídios no Brasil. São Paulo: Instituto Sangari; 2010.

40. Wang F, Arnold MT. Localized income inequality, concentrated disadvantage and homicide. Appl Geogr. 2008;9(4):259-70.

41. Nery MB, Monteiro AMV. Análise intraurbana dos homicídios dolosos no município de São Paulo. XIV Encontro Nacional de Estudos Populacionais, ABEP. Caxambu; 2006. Disponível em: http://www.abep.nepo.unicamp. br/encontro2006/docspdf/ABEP2006_278. pdf. Acessado em 22 de novembro de 2012.

42. Dias TL, Oliveira MPG, Câmara G, Carvalho MS. Problemas de escala e a relação áreaindivíduo em análise espacial de dados censitários. Informática Pública. 2002;4(1):89-104.
43. Greenland S, Morgenstern H. Ecological bias, confounding, and effect modification. Int J Epidemiol. 1989;18(1):269-74.

44. Susser M. The logic of ecological: I. The logic of analysis. Am J Public Health. 1994;84(5):825-9.

45. Wrigley N, Holt T, Steel D, Trammer M. Analysing, modelling, and resolving the ecological fallacy. Em: Longley P, Batty M, eds. Spatial analysis: modelling in a GIS environment. Nova Iorque: John Wiley \& Sons; 1996. Pp. 25-41.

46. Câmara G, Carvalho MS, Cruz OG, Correa V. Análise espacial de áreas. São José dos Campos: INPE; 2004.

47. Santos SM, Barcellos C, Carvalho SM. Ecological analysis of the distribution and social- -spatial context of homicides in Porto Alegre, Brazil. Health Place. 2006;12(1):38-47.

48. Rex J. Problemas fundamentais da teoria sociológica. Rio de Janeiro: Zahar; 1973.

49. Da Cunha MEC, Da Silva JMP. O uso do território e as relações de Poder no Município de Barcarena-PA. Rev Cientifica UFPA. 2007;6(1).

Manuscrito recebido em 7 de dezembro de 2011. Aceito em versão revisada em 9 de setembro de 2012.

ABSTRACT Objective. To identify the existence of spatial and temporal patterns in the occurrence of intentional homicides in the municipality of São Paulo (MSP), Brazil, and to

\section{Spatial regimes: dynamics of intentional homicides in the city of São Paulo between 2000 and 2008}

discuss the analytical value of taking such patterns into account when designing studies that address the dynamics and factors associated with the incidence of homicides. Methods. A longitudinal ecological study was conducted, having as units of analysis 13205 census tracts and the 96 census districts that congregate these sectors in São Paulo. All intentional homicides reported in the city between 2000 and 2008 were analyzed. The gross homicide rates per 100000 population was calculated as well as the global and local Bayesian estimates for each census tract during the study period. To verify the possibility of identifying different patterns of the spatial distribution of homicides, we used BoxMap and Moran's I index.

Results. The homicide trends in the city of São Paulo in the last decade were not homogeneous and systematic. Instead, seven patterns of spatial distribution were identified; that is, seven spatial regimes for the occurrence of intentional homicides, considering the homicide rates within each census tract as well as the rates in adjacent tracts. These spatial distribution regimes were not contained within the limits of the census tracts and districts.

Conclusions. The results show the importance of analyzing the spatial distribution of social phenomena without restriction of political and administrative boundaries.

Key words Homicide; residence characteristics; temporal distribution; geographic information systems; Brazil. 